\title{
Modelagem para o dimensionamento de microtubos em irrigação localizada
}

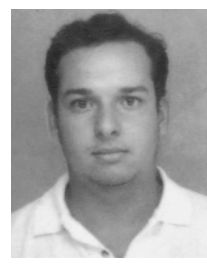

Rodrigo O. R. de M. Souza'1 \& Tarlei A. Botrel²

1 USP/ESALQ. Av. Pádua Dias 11, CEP 13418-900, Piracicaba, SP. Fone: (19) 3429-4217. E-mail: rormsouz@esalq.usp.br (Foto)

2 USP/ESALQ. Fone: (19) 3429-4217, Ramal: 255. E-mail: tabotrel@esalq.usp.br

Protocolo 66 - 27/6/2003 - Aprovado em 16/12/2003

\begin{abstract}
Resumo: Com o intuito de oferecer embasamento técnico-científico para o desenvolvimento de uma irrigação de baixo custo e alto nível tecnológico, este trabalho teve como objetivo desenvolver uma equação para dimensionamento de microtubos em que a perda de carga localizada, a perda de carga ao longo do tubo e a energia de velocidade, estão representados na equação. O presente trabalho foi conduzido no Departamento de Engenharia Rural da ESALQ/USP, onde foi estabelecida, com base nos resultados dos ensaios dos microtubos, a relação entre pressão, comprimento, vazão e diâmetro para três diferentes modelos matemáticos de representação do fenômeno de perda de energia no microtubo. Dentre os modelos propostos, o que considerou a perda de carga localizada nesse fenômeno, com o coeficiente K da equação de perda carga localizada em função do Número de Reynolds (RN) foi o que melhor representou o fenômeno de perda de energia no microtubo. Foi testado, também, um modelo que não considera a perda de carga localizada e um outro que ressalta a perda de carga localizada com o coeficiente de perda de carga localizada (K) constante. A perda de carga localizada é um importante fator que deve ser considerado no dimensionamento do microtubo. Além disso, em situações de baixo NR o coeficiente de perda de carga localizada (K) não é um valor constante e, sim, uma função do NR.
\end{abstract}

Palavras-chave: gotejamento, hidráulica, perda de carga

\section{Modeling for the design of microtubes in trickle irrigation}

\begin{abstract}
The objective of this work was to develop an equation for the design of microtubes where the friction head loss in the microtube wall, the localized head loss and the velocity are explicit in the equation. The aim of this work was to offer a technical base for the development of a high irrigation technology with low cost. The work was carried out at the Rural Engineering Department of the ESALQ/USP. Based on the results of microtube tests the relation between pressure, length, flow and diameter for three different models of microtube head loss were established. The best representation of the microtube head loss was given by the model which considered the localized head loss with the coefficient $\mathrm{K}$ of the localized head loss equation depending on the Reynolds Number (RN). The other models tested were the model without localized head loss and the one considering the localized head loss with a constant $\mathrm{K}$. The localized head loss is an important parameter which should be considered in the design of microtubes. Besides this, the coefficient of the localized head loss $(\mathrm{K})$ is not a constant value but is a function of RN.
\end{abstract}

Key words: localized irrigation, hydraulics, microtubes

\section{INTRODUÇÃO}

O microtubo é o mais antigo gotejador de longo percurso. Esse emissor nada mais é que um pequeno tubo de plástico com diâmetro entre 0,5 e $2 \mathrm{~mm}$, que se caracteriza pela fácil instalação e baixo custo, quando comparado com outros tipos de emissores.

O microtubo, quando utilizado, dissipa energia ao longo de seu comprimento, liberando uma quantidade pequena de água para irrigação. O seu comprimento e diâmetro podem ser selecionados para diferentes vazões, possibilitando utilizá-lo como emissor. Segundo Silva et al. (1988), considerando o processo artesanal e o baixo custo de confecção/instalação, linhas laterais com emissores do tipo microtubo de tamanhos variados, quando bem dimensionados, podem ser utilizados em sistemas localizados, obtendo-se razoáveis níveis de uniformidade de distribuição de água.

A utilização de microtubos vem crescendo especialmente em países pobres da Ásia e África, onde organizações internacionais lutam pelo desenvolvimento dos pequenos agricultores através da implantação de tecnologias de baixo custo. Instituições, como International Development Enterprises (IDE) 
e Intermediate Technology Consultants (ITC), promovem na Índia e no Zimbábue a utilização da irrigação por microtubos com alimentação por gravidade. Linhas laterais, microtubos e baldes são utilizados como ferramentas que proporcionam o desenvolvimento da população pobre no meio rural (Mackay, 2003).

O principal item no dimensionamento de microtubos é o cálculo da perda de carga causada pelo emissor em determinada condição de vazão e pressão. Conforme Khatri et al. (1979) e Ishaq \& Ibrahim (1995), esta perda de energia é uma combinação entre perda de carga na parede do tubo e perdas menores. Essas perdas menores correspondem à perda de carga localizada e à energia de velocidade.

Os microtubos apresentam, quando trabalham em regime laminar, os inconvenientes de alta sensibilidade a variações de temperatura e pressão, além de maiores riscos de entupimento (Soares, 1981). Mesmo com essas dificuldades, Pizarro Cabello (1987) comenta que na Espanha os microtubos se tornaram muito populares pelo seu baixo custo e por uma peculiaridade interessante: o agricultor pode uniformizar as vazões, mesmo o sistema estando instalado, cortando os emissores no comprimento que se mostre adequado.

Um entrave para o desenvolvimento dessa tecnologia, além da sensibilidade ao entupimento e a temperatura, é a necessidade de um projeto criterioso e detalhado, de forma que a vazão do microtubo seja estimada com bastante precisão.

Para que a vazão do microtubo seja estimada com precisão é necessário que se utilize uma equação que correlacione vazão, comprimento, pressão na entrada do microtubo e diâmetro.

$\mathrm{Na}$ literatura existem equações empíricas que expressam esta relação, porém são equações que não discriminam perda de carga localizada, perda de carga na parede do tubo nem energia de velocidade (Eq. 1, 2 e 3).

$$
\begin{gathered}
\text { Hftubo }=\mathrm{f} \frac{\mathrm{L}}{\phi} \frac{\mathrm{V}^{2}}{2 \mathrm{~g}} \\
\text { Hflocalizado }=\mathrm{K} \frac{\mathrm{V}^{2}}{2 \mathrm{~g}} \\
\mathrm{EV}=\frac{\mathrm{V}^{2}}{2 \mathrm{~g}}
\end{gathered}
$$

em que:

Hftubo - perda de carga na parede do tubo (m)

f - fator de atrito da fórmula universal (adimensional)

L - comprimento do microtubo (m)

$\phi \quad$ - diâmetro do microtubo (m)

$\mathrm{V}$ - velocidade da água no microtubo $\left(\mathrm{m} \mathrm{s}^{-1}\right)$

g - aceleração da gravidade $\left(\mathrm{m} \mathrm{s}^{-2}\right)$

Hflocalizado - perda de carga localizada (m)

K - coeficiente de perda de carga localizada (adimensional)

Ev - energia de velocidade (m)

Khatri et al. (1979) realizaram um estudo sobre hidráulica de microtubos e determinaram equações que representam a perda de carga total em diversas condições de regime de escoamento (Eq. 4, 5, 6 e 7). Os referidos autores consideram neste trabalho, que as perdas localizadas podem ser negligenciadas no regime laminar.

- Equação geral para qualquer regime:

$$
\mathrm{H}=0,0054 \frac{\mathrm{Q}^{1,516}}{\phi^{4,245}} \mathrm{~L}
$$

- Equação para regime laminar:

$$
\mathrm{H}=0,0074 \frac{\mathrm{Q}^{1,253}}{\phi^{3,361}} \mathrm{~L}
$$

- Equação para regime de transição:

$$
\mathrm{H}=0,0034 \frac{\mathrm{Q}^{1,665}}{\phi^{4,190}} \mathrm{~L}
$$

- Equação para regime turbulento:

$$
\mathrm{H}=0,0036 \frac{\mathrm{Q}^{1,779}}{\phi^{4,857}} \mathrm{~L}
$$

em que:

$$
\begin{array}{ll}
\mathrm{H} & \text { - perda de carga total }(\mathrm{m}) \\
\phi & \text { - diâmetro do microtubo }(\mathrm{mm}) \\
\mathrm{Q} & \text { - vazão do emissor }\left(\mathrm{L} \mathrm{h}^{-1}\right) \\
\mathrm{L} & \text { - comprimento do microtubo }(\mathrm{cm})
\end{array}
$$

Segundo Vermeiren \& Jobling (1980) os microtubos possuem grandes vantagens, são utilizados em inúmeros países e adaptam-se a parcelas com declividade acentuada e variada. Segundo os mesmos autores a relação entre vazão, pressão de serviço, comprimento e diâmetro interno, pode ser expressa por:

$$
\mathrm{q}=\mathrm{aL}^{\mathrm{b}} \mathrm{H}^{\mathrm{c}} \phi^{\mathrm{d}}
$$

em que:

$$
\begin{array}{ll}
\mathrm{q} & \text { - vazão do microtubo }\left(\mathrm{L} \mathrm{h}^{-1}\right) \\
\mathrm{L} & \text { - comprimento do microtubo }(\mathrm{m}) \\
\mathrm{H} & \text { - pressão de serviço }(\mathrm{m}) \\
\phi & \text { - diâmetro do microtubo }(\mathrm{mm})
\end{array}
$$

a, b, c e d - coeficientes que dependem do diâmetro (Vermeiren \& Jobling, 1980)

Uma dificuldade na elaboração de projetos com microtubo é a atribuição de seu diâmetro para dimensionamento. Em virtude dos pequenos diâmetros, qualquer erro na sua atribuição pode provocar variação na estimativa da vazão. Mesmo que o projetista tenha em mãos uma equação empírica desenvolvida por um criterioso trabalho científico, não é 
garantido que esta equação estimará com precisão a vazão do microtubo. $\mathrm{O}$ diâmetro fornecido pelo fabricante pode não representar com precisão o diâmetro real.

Em virtude dessas dificuldades, Pizarro Cabello (1987) recomenda que as características dos microtubos sejam determinadas experimentalmente; entretanto, não adianta realizar somente ensaios de laboratório mas é importante, também, utilizar um modelo adequado de representação do fenômeno de perda de energia no microtubo, coerente com os fundamentos básicos de hidráulica.

Dentro desse contexto e com o intuito de oferecer embasamento técnico-científico para o desenvolvimento de uma irrigação de baixo custo e alto nível tecnológico, este trabalho teve como objetivo desenvolver uma equação para dimensionamento de microtubos em que a perda de carga localizada, a perda de carga ao longo do tubo e a energia de velocidade, estarão explícitos na equação.

\section{MATERIAL E MÉTODOS}

O presente trabalho foi conduzido no Laboratório de Hidráulica do Departamento de Engenharia Rural da ESALQ/ USP, onde foi estabelecida com base nos resultados dos ensaios dos microtubos, a relação entre pressão, comprimento, vazão e diâmetro para três diferentes modelos matemáticos de representação do fenômeno de perda de energia no microtubo.

A avaliação dos microtubos foi realizada em diferentes condições de diâmetro, pressão e comprimento. Durante o ensaio os emissores funcionaram em regime laminar, situação esta considerada na elaboração dos três modelos, com o intuito de evitar as incertezas e as dificuldades de representação do fenômeno de perda de energia no microtubo em regime de transição.

Foram avaliados microtubos com diâmetros internos de 1 $\mathrm{mm}$ (A), 0,8 mm (B), 0,7 mm (C) e 0,6 mm (D). Os valores dos diâmetros foram fornecidos pelo fabricante.

Os microtubos foram ensaiados nos comprimentos de 0,1 a 1,5 $\mathrm{m}$ (com incrementos de $0,1 \mathrm{~m}$ ). Para cada comprimento o emissor foi posto em funcionamento em cinco ou seis pressões de serviço diferentes. A pressão de serviço do emissor foi regulada através de um registro de precisão e medida com um manômetro de mercúrio. A vazão do microtubo foi mensurada três vezes em cada condição de pressão. As pressões em que foram realizados os ensaios nos respectivos comprimentos encontram-se na Tabela 1, cujos valores foram selecionados na fase de planejamento do trabalho; durante os ensaios foram realizadas medidas de vazão com pressões próximas a esses valores.

Os microtubos foram inseridos em um tubo de polietileno de $13 \mathrm{~mm}$. A inserção do microtubo no tubo de $13 \mathrm{~mm}$ foi padronizada em $5 \mathrm{~mm}$. A vazão dos emissores foi calculada com base no volume de água coletado durante o tempo de três minutos. O esquema da bancada de ensaios pode ser visto na Figura 1.

Foram testados três modelos de representação do fenômeno de perda de energia no microtubo, em que os resultados dos ensaios de laboratório foram utilizados para determinação dos coeficientes desses modelos. Esses coeficientes, por sua vez, foram obtidos pelo software computacional Table Curve 3D.

O Modelo 1 é uma representação do fenômeno de perda de energia no microtubo, sem considerar a perda de carga localizada, enquanto o Modelo 2 considera a perda de carga localizada com o coeficiente K da Eq. 2 constante; já o modelo 3 também ressalta a presença da perda de carga localizada; entretanto, com coeficiente K da Eq. 2 em função do Número de Reynolds (NR) (Eq. 9, 10 e 11).

Tabela 1. Valores de pressões e comprimentos de microtubo em que foram realizadas medidas de vazão

\begin{tabular}{cccccccc}
\hline $\mathrm{L}(\mathrm{m})$ & \multicolumn{7}{c}{ Pressões $(\mathrm{kPa})$} \\
\hline 0,1 & 1,5 & 3,0 & 4,5 & 6,0 & 7,5 & 9,0 & \\
0,2 & 3,0 & 7,0 & 10,0 & 13,0 & 16,0 & 19,0 & \\
0,3 & 5,0 & 10,0 & 15,0 & 20,0 & 25,0 & 30,0 & \\
0,4 & 6,0 & 12,0 & 18,0 & 24,0 & 30,0 & 36,0 & \\
0,5 & 8,0 & 16,0 & 24,0 & 32,0 & 36,0 & 40,0 & 46,0 \\
0,6 & 10,0 & 20,0 & 30,0 & 40,0 & 50,0 & 60,0 & \\
0,7 & 10,0 & 20,0 & 30,0 & 40,0 & 50,0 & 60,0 & 70,0 \\
0,8 & 13,0 & 26,0 & 39,0 & 52,0 & 65,0 & 78,0 & \\
0,9 & 15,0 & 30,0 & 45,0 & 60,0 & 75,0 & 90,0 & \\
1,0 & 15,0 & 30,0 & 45,0 & 60,0 & 75,0 & 90,0 & \\
1,1 & 15,0 & 30,0 & 45,0 & 60,0 & 75,0 & 90,0 & 100,0 \\
1,2 & 20,0 & 40,0 & 60,0 & 80,0 & 100,0 & 120,0 & \\
1,3 & 20,0 & 40,0 & 60,0 & 80,0 & 100,0 & 120,0 & \\
1,4 & 20,0 & 40,0 & 60,0 & 80,0 & 100,0 & 120,0 & 130,0 \\
1,5 & 25,0 & 50,0 & 75,0 & 100,0 & 125,0 & 150,0 & \\
\hline
\end{tabular}

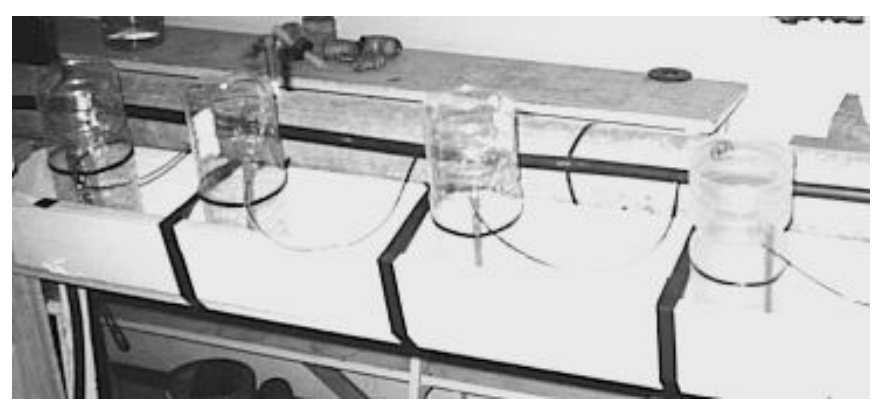

Figura 1. Bancada para ensaios de laboratório

- Modelo 1: $\mathrm{H}=$ Hftubo $+\mathrm{EV}$

$$
\mathrm{H}=\frac{64}{\mathrm{NR}} \frac{\mathrm{L}}{\phi} \frac{\mathrm{V}^{2}}{2 \mathrm{~g}}+\frac{\mathrm{V}^{2}}{2 \mathrm{~g}}
$$

em que:

H - pressão na entrada do tubo (m)

$\mathrm{NR}$ - número de Reynolds (adimensional)

L - comprimento do microtubo (m)

$\mathrm{V}$ - velocidade da água no microtubo $\left(\mathrm{m} \mathrm{s}^{-1}\right)$

g - aceleração da gravidade $\left(\mathrm{m} \mathrm{s}^{-2}\right)$

$\phi \quad$ - diâmetro do microtubo (m)

- Modelo 2: H = Hftubo + EV + Hflocalizado

$$
\mathrm{H}=\frac{64}{\mathrm{NR}} \frac{\mathrm{L}}{\phi} \frac{\mathrm{V}^{2}}{2 \mathrm{~g}}+\frac{\mathrm{V}^{2}}{2 \mathrm{~g}}+\mathrm{K} \frac{\mathrm{V}^{2}}{2 \mathrm{~g}}
$$

em que:

K - coeficiente da equação de perda de carga localizada 
- Modelo 3: $\mathrm{H}=$ Hftubo $+\mathrm{EV}+$ Hflocalizado

$$
\mathrm{H}=\frac{64}{\mathrm{NR}} \frac{\mathrm{L}}{\phi} \frac{\mathrm{V}^{2}}{2 \mathrm{~g}}+\frac{\mathrm{V}^{2}}{2 \mathrm{~g}}+(\mathrm{a} \ln (\mathrm{NR})+\mathrm{b}) \frac{\mathrm{V}^{2}}{2 \mathrm{~g}}
$$

em que: "a" e "b"- coeficientes da equação que expressa K em
função do NR (adimensional)

Nos três modelos a perda de carga na parede do tubo é calculada com a fórmula universal, considerando-se o regime laminar; logo, o fator de atrito da fórmula universal é calculado pela Eq. 12. Os três modelos consideram também a energia de velocidade no fenômeno de perda de energia no microtubo.

$$
f=\frac{64}{N R}
$$

Para amenizar o erro na estimativa do diâmetro, foi obtido um valor de diâmetro interno para cada tipo de microtubo. Este diâmetro, determinado com base no resultado dos ensaios, foi nomeado de diâmetro efetivo.

Com base nos ensaios de laboratório e no Modelo 1 (Eq. 9) obteve-se através do Table Curve 3D, um diâmetro efetivo para cada tipo de microtubo (A, B, C e D). Com os mesmos dados de laboratório e a equação do Modelo 2 (Eq. 10), além dos diâmetros efetivos, foram determinados também os coeficientes de perda de carga localizada $(\mathrm{K})$ para cada tipo de microtubo. $\mathrm{O}$ mesmo procedimento foi repetido com a equação do Modelo 3 (Eq. 11) para obtenção dos diâmetros efetivos e os coeficientes "a" e "b" da relação entre K (coeficiente de perda de carga localizada) e NR para cada tipo de microtubo.

Para escolha do modelo que melhor representa a perda de energia no microtubo, foram estimados valores de pressão para as diversas condições de vazão, comprimento e diâmetro efetivo. Esses valores foram comparados com os valores obtidos em laboratório. Os valores estimados de diâmetro efetivo também foram comparados com os valores de diâmetro medidos em dez unidades de cada microtubo.

Também foram estimados valores de pressão para as diversas condições de vazão, comprimento e diâmetro efetivo com as equações propostas por Vermeiren \& Jobling (1980) e Khatri et al. (1979). O diâmetro efetivo utilizado nessa estimativa foi o diâmetro resultante do ajuste do modelo que obteve o melhor desempenho dentre os modelos propostos.

Outro parâmetro de comparação entre modelos foi o Erro Médio Absoluto (EMA). Para obtenção do EMA calcula-se a diferença entre o valor estimado de pressão e o valor medido. O EMA de cada modelo é a média entre os valores absolutos dessas diferenças.

\section{RESULTADOS E DISCUSSÃO}

\section{Ensaios de laboratório}

Os resultados dos ensaios de laboratório podem ser vistos na Figura 2 e foram expressos graficamente pelas curvas vazão versus pressão, correspondente a cada comprimento de microtubo em seus diversos diâmetros.

Durante o ensaio não foram constatados vazamentos na inserção do microtubo na linha lateral, demonstrando que a introdução direta do microtubo na linha é uma alternativa viável de montagem do sistema.

Com o projetor de perfil automático e se usando uma ampliação de cinqüenta vezes, mediram-se os diâmetros de dez amostras de cada modelo de microtubo (Tabela 2). O microtubo $\mathrm{D}$ apresentou uma diferença de $0,0794 \mathrm{~mm}(13,2 \%)$ entre o valor médio medido e o dado de catálogo. Os microtubos A, B e C, apresentaram diferenças de 2,4,2,5 e 0,7\%, respectivamente. Mesmo o valor do diâmetro estando próximo do valor de catálogo, em se tratando de microtubo, uma diferença de 0,05 $\mathrm{mm}$ no valor do diâmetro poder provocar uma estimativa errônea da vazão, o que comprova a importância da determinação de um diâmetro efetivo em laboratório que possa ser utilizado posteriormente no dimensionamento.

\begin{tabular}{|c|c|c|c|c|}
\hline \multirow{2}{*}{ Microtubo } & A & $\mathrm{B}$ & $\mathrm{C}$ & $\mathrm{D}$ \\
\hline & \multicolumn{4}{|c|}{ Diâmetro (mm) } \\
\hline 1 & 0,999 & 0,811 & 0,718 & 0,654 \\
\hline 2 & 0,998 & 0,831 & 0,696 & 0,636 \\
\hline 3 & 0,995 & 0,817 & 0,708 & 0,701 \\
\hline 4 & 0,999 & 0,820 & 0,702 & 0,690 \\
\hline 5 & 0,962 & 0,833 & 0,681 & 0,692 \\
\hline 6 & 1,037 & 0,811 & 0,683 & 0,685 \\
\hline 7 & 1,091 & 0,840 & 0,666 & 0,675 \\
\hline 8 & 1,062 & 0,822 & 0,691 & 0,697 \\
\hline 9 & 1,061 & 0,787 & 0,691 & 0,691 \\
\hline 10 & 1,036 & 0,830 & 0,713 & 0,673 \\
\hline Média & 1,024 & 0,820 & 0,695 & 0,679 \\
\hline Diferença (\%) & 2,4 & 2,5 & 0,7 & 13,2 \\
\hline
\end{tabular}

Tabela 2. Diâmetros dos microtubos medidos com o perfilômetro

\section{Modelos propostos}

O Modelo 1 está representado pela Eq. 13. Foi realizado um ajuste da Eq. 13, através do Table Curve 3D, para obtenção do diâmetro efetivo de cada microtubo. O software ajustou o valor de diâmetro efetivo para as diversas condições de pressão, vazão e comprimento obtidos em laboratório.

$$
\mathrm{H}=\frac{64 \mathrm{v} 4}{\pi 2 \mathrm{~g}}\left(\frac{\mathrm{LQ}}{\phi^{4}}\right)+\frac{16}{\pi^{2} 2 \mathrm{~g}}\left(\frac{\mathrm{Q}^{2}}{\phi^{4}}\right)
$$

em que:

V - viscosidade cinemática da água $\left(\mathrm{m}^{2} \mathrm{~s}^{-1}\right)$

Q - vazão do microtubo $\left(\mathrm{m}^{3} \mathrm{~s}^{-1}\right)$

Os valores de diâmetro efetivo para cada microtubo e o coeficiente de determinação do ajuste obtidos com o Modelo 1, estão na Tabela 3.

O Modelo 2 está representado pela Eq. 14. Foi realizado um ajuste da Eq. 14, através do Table Curve 3D, para obtenção do diâmetro efetivo e do coeficiente de perda de carga localizada (K) de cada microtubo. O software ajustou os valores de diâmetro efetivo e $\mathrm{K}$ para as diversas condições de pressão, vazão e comprimento obtidos em laboratório. 

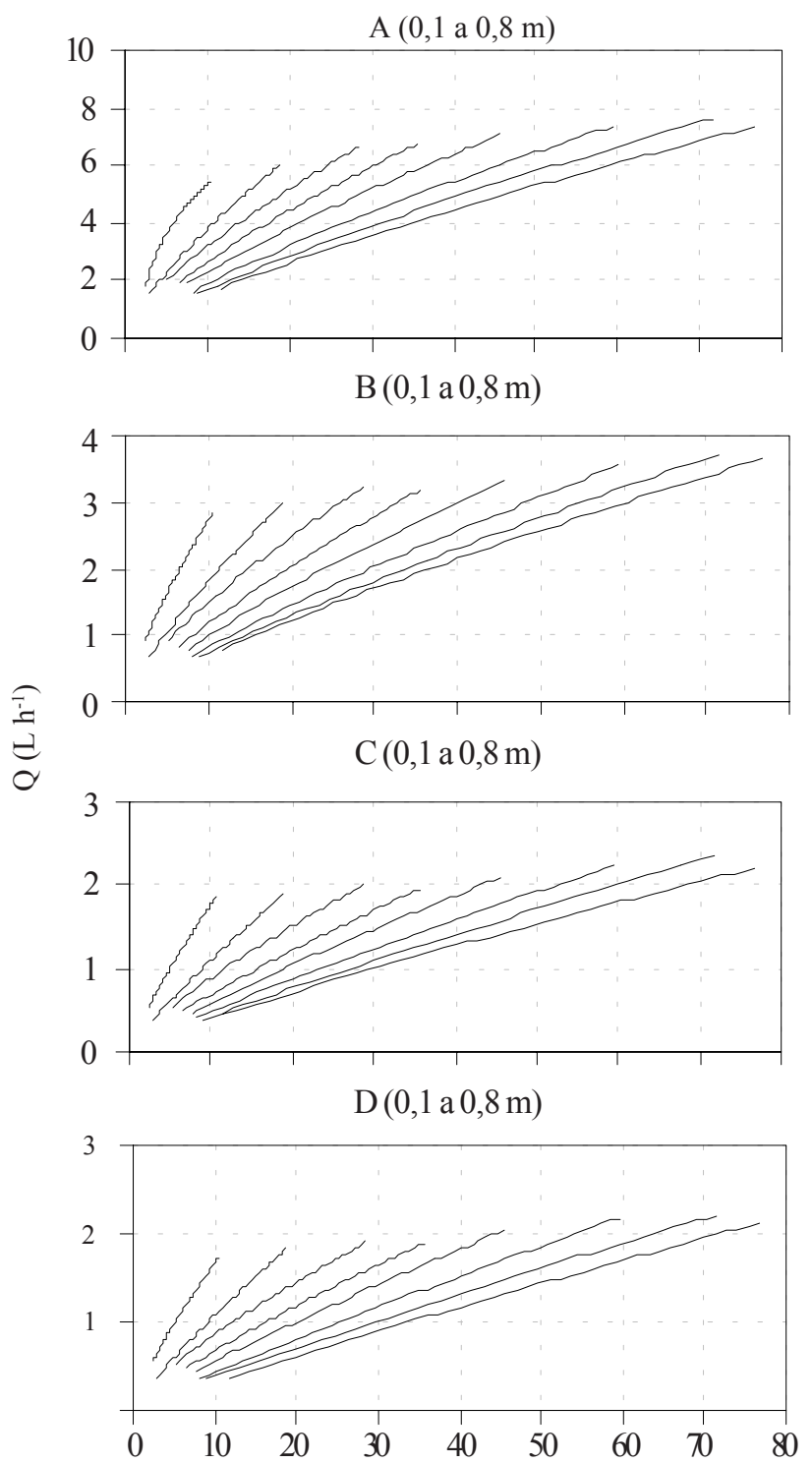

A $(0,9$ a $1,5 \mathrm{~m})$
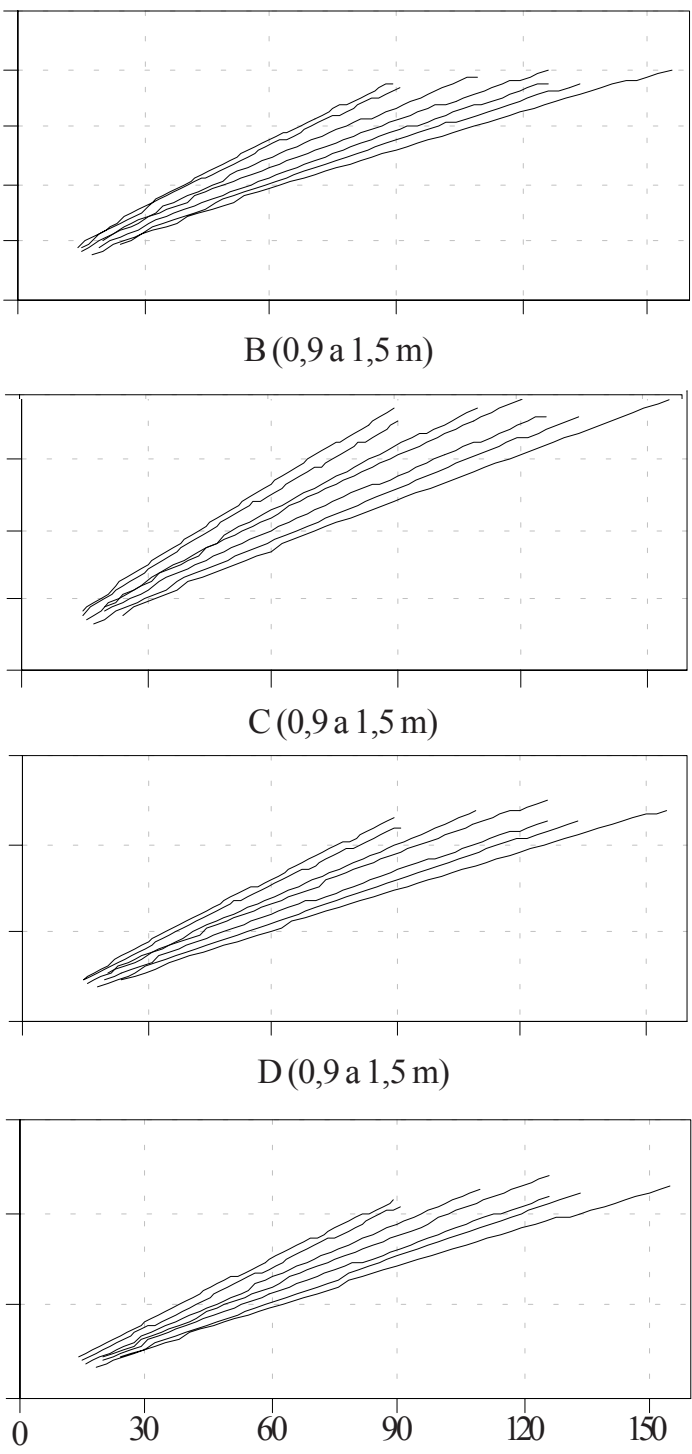

$\mathrm{H}(\mathrm{kPa})$

Figura 2. Curvas vazão versus pressão dos diversos comprimentos de microtubo para os quatro diâmetros

Tabela 3. Valores de diâmetro efetivo para cada microtubo e coeficiente de determinação do ajuste obtidos com o modelo 1

\begin{tabular}{ccc} 
Microtubo & $\mathrm{r}^{2}$ & Diâmetro Efetivo $(\mathrm{mm})$ \\
\hline A & 0,976 & 0,994 \\
B & 0,988 & 0,823 \\
C & 0,991 & 0,728 \\
D & 0,994 & 0,717 \\
\hline
\end{tabular}

$$
\mathrm{H}=\frac{64 \mathrm{v} 4}{\pi 2 \mathrm{~g}}\left(\frac{\mathrm{LQ}}{\phi^{4}}\right)+\frac{16}{\pi^{2} 2 \mathrm{~g}}\left(\frac{\mathrm{Q}^{2}}{\phi^{4}}\right)+\frac{16}{\pi^{2} 2 \mathrm{~g}}\left(\frac{\mathrm{KQ}^{2}}{\phi^{4}}\right)
$$

Os valores de diâmetro efetivo, K e o coeficiente de determinação do ajuste para cada microtubo, obtidos com o modelo 2 , estão na Tabela 4.

No Modelo 3, está representado pela Eq. 15. Realizou-se um ajuste da Eq. 15 através do Table Curve 3D, para obtenção do diâmetro efetivo e dos coeficientes da equação de $\mathrm{K}$ em função do NR ("a" e "b") de cada microtubo. O software ajustou
Tabela 4. Valores de diâmetro efetivo, K e coeficiente de determinação do ajuste obtidos com o Modelo 2

\begin{tabular}{cccc} 
Microtubo & $\mathrm{r}^{2}$ & $\mathrm{~K}$ & Diâmetro Efetivo $(\mathrm{mm})$ \\
\hline A & 0,980 & 3,153 & 1,018 \\
B & 0,992 & 5,302 & 0,839 \\
C & 0,994 & 8,164 & 0,742 \\
D & 0.996 & 6,963 & 0,729 \\
\hline
\end{tabular}

os valores de diâmetro efetivo, "a" e "b" para as diversas condições de pressão, vazão e comprimento obtidos em laboratório.

$$
\mathrm{H}=\frac{64 \mathrm{v} 4}{\pi 2 \mathrm{~g}}\left(\frac{\mathrm{LQ}}{\phi^{4}}\right)+\frac{16}{\pi^{2} 2 \mathrm{~g}}\left(\frac{\mathrm{Q}^{2}}{\phi^{4}}\right)+\left(\frac{(\mathrm{a} \ln (\mathrm{NR})+\mathrm{b}) \mathrm{Q}^{2}}{\phi^{4}}\right)
$$

Os valores de diâmetro efetivo, "a", "b" e o coeficiente de determinação do ajuste para cada microtubo obtidos com o Modelo 3, encontram-se na Tabela 5. 
Tabela 5. Valores de diâmetro efetivo, "a", "b" e coeficiente de determinação do ajuste obtidos com o modelo 3

\begin{tabular}{ccccc}
\hline Microtubo & $\mathrm{r}^{2}$ & $\mathrm{a}$ & $\mathrm{b}$ & Diâmetro Efetivo $(\mathrm{mm})$ \\
\hline $\mathrm{A}$ & 0,994 & 1,007 & $-7,584$ & 1,009 \\
$\mathrm{~B}$ & 0,997 & 1,154 & $-7,959$ & 0,835 \\
$\mathrm{C}$ & 0,997 & 1,533 & $-9,926$ & 0,738 \\
$\mathrm{D}$ & 0,998 & 1,401 & $-9,062$ & 0,726 \\
\hline
\end{tabular}

Com os valores de vazão, comprimento e diâmetro efetivo do microtubo, foram estimados valores de pressão. Este procedimento foi repetido com cada modelo em estudo. Os valores estimados de pressão foram comparados com os valores obtidos em laboratório (Figura 3). A comparação foi realizada graficamente sendo que, quanto mais a equação ajustada (pressão estimada versus pressão medida) estiver próxima da equação $\mathrm{x}=\mathrm{y}$, mais os valores estimados se aproximam dos valores medidos, além disso, quanto maior o valor do $\mathrm{r}^{2}$ menor será a dispersão dos pontos em relação à equação ajustada.

Todos os modelos obtiveram valores de diâmetro efetivo próximos do valor médio medido. A diferença em cada microtubo entre o diâmetro efetivo e o diâmetro medido, em porcentagem, encontra-se na Tabela 6.

Embora os três modelos tenham obtido equações de pressão medida versus pressão estimada próximas da reta $\mathrm{x}=\mathrm{y}$ com bom ajuste $\left(\mathrm{r}^{2}\right)$, os valores de erro médio absoluto demonstram que o Modelo 3 teve melhor desempenho (Tabela 6).

O melhor ajuste do Modelo 3 demonstra que é importante considerar a perda de carga localizada no fenômeno de perda de energia no microtubo. Além disso, é imprescindível considerar que o coeficiente de perda de carga localizada $(\mathrm{K})$ não é um valor constante em situações de baixo NR e, sim, uma função do NR. Segundo Porto (1999) o valor de K tende a ficar constante em situações com altos valores de NR.

Recomenda-se a utilização deste modelo para o dimensionamento de outros microtubos, desde que os parâmetros do modelo sejam previamente ajustados e que o regime de escoamento no microtubo seja laminar.

Equações de Khatri et al. (1979) e Vermeiren \& Jobling (1980)

Também foram estimados valores de pressão para as diversas condições de vazão, comprimento e diâmetro efetivo, com as equações propostas por Khatri et al. (1979) e Vermeiren \& Jobling (1980) (Eq. 5 e 8). O diâmetro efetivo utilizado nessa
A. Modelo 1
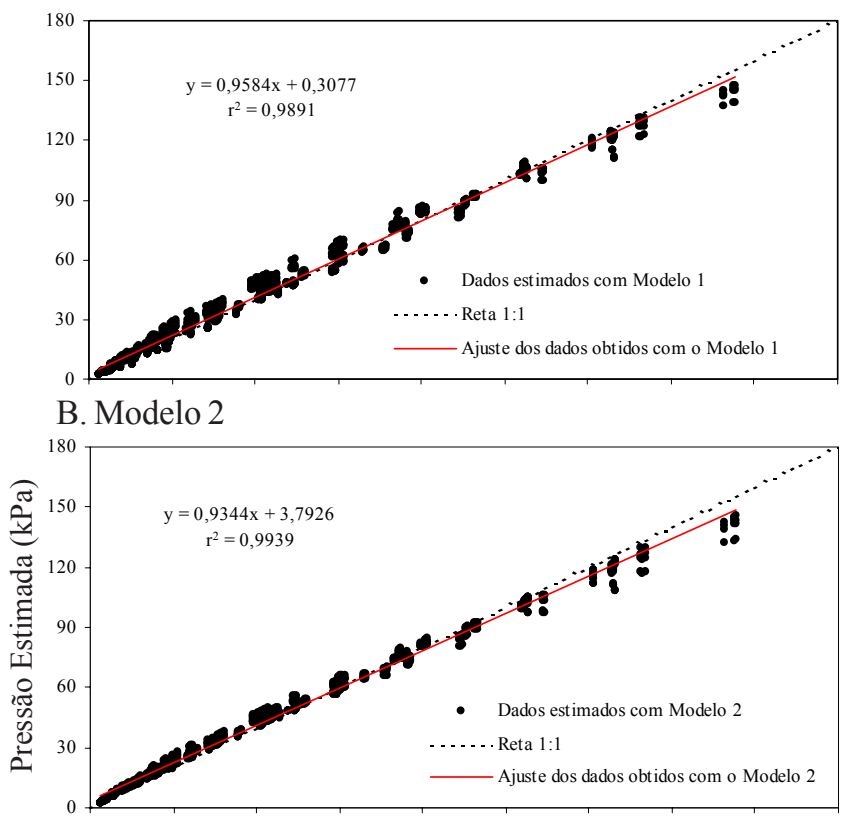

C. Modelo 3

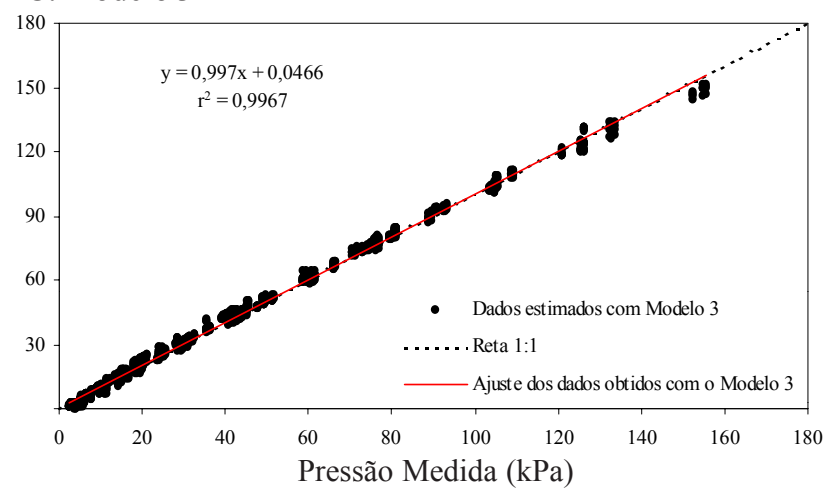

Figura 3. Relações entre pressões medidas e pressões estimadas, obtidas com base nos três modelos propostos

estimativa foi o diâmetro obtido com o Modelo 3 que, dentre os modelos propostos, foi o que obteve o melhor desempenho na estimativa do valor de pressão (Figura 4).

A equação proposta por Vermeiren \& Jobling (1980) obteve uma função pressão medida versus pressão estimada próxima da reta $\mathrm{x}=\mathrm{y}$ com um $\mathrm{r}^{2}$ de 0,959 ; já a equação proposta por Khatri et al. (1979), que resultou em um $r^{2}$ de 0,8965, não obteve

Tabela 6. Parâmetros obtidos com base nos modelos propostos

\begin{tabular}{|c|c|c|c|c|c|c|c|}
\hline \multirow{2}{*}{ Modelos } & \multirow{2}{*}{ Microtubo } & \multicolumn{2}{|c|}{ Diâmetro (mm) } & \multirow{2}{*}{$\begin{array}{l}\text { Diferença } \\
(\%)\end{array}$} & \multirow{2}{*}{$\begin{array}{l}\text { Pressão Medida versus } \\
\text { Pressão Estimada }\end{array}$} & \multirow{2}{*}{$r^{2}$} & \multirow{2}{*}{$\begin{array}{l}\text { EMA } \\
(\mathrm{m})\end{array}$} \\
\hline & & Efetivo & Medido & & & & \\
\hline \multirow{4}{*}{ Modelo 1} & A & 0,994 & 1,024 & 2,90 & \multirow{4}{*}{$y=0,9584 x+3,077$} & \multirow{4}{*}{0,9891} & \multirow{4}{*}{0,31} \\
\hline & B & 0,823 & 0,820 & 0,36 & & & \\
\hline & C & 0,728 & 0,695 & 4,75 & & & \\
\hline & $\mathrm{D}$ & 0,717 & 0,679 & 5,59 & & & \\
\hline \multirow{4}{*}{ Modelo 2} & A & 1,018 & 1,024 & 0,58 & \multirow{4}{*}{$y=0,9934 x+3,793$} & \multirow{4}{*}{0,9948} & \multirow{4}{*}{0,28} \\
\hline & B & 0,839 & 0,820 & 2,30 & & & \\
\hline & C & 0,742 & 0,695 & 6,76 & & & \\
\hline & $\mathrm{D}$ & 0,729 & 0,679 & 7,36 & & & \\
\hline \multirow{4}{*}{ Modelo 3} & A & 1,009 & 1,024 & 1,46 & \multirow{4}{*}{$Y=0,997 x+0,466$} & \multirow{4}{*}{0,9967} & \multirow{4}{*}{0,16} \\
\hline & B & 0,835 & 0,820 & 1,83 & & & \\
\hline & $\mathrm{C}$ & 0,738 & 0,695 & 6,18 & & & \\
\hline & D & 0,726 & 0,679 & 6,92 & & & \\
\hline
\end{tabular}




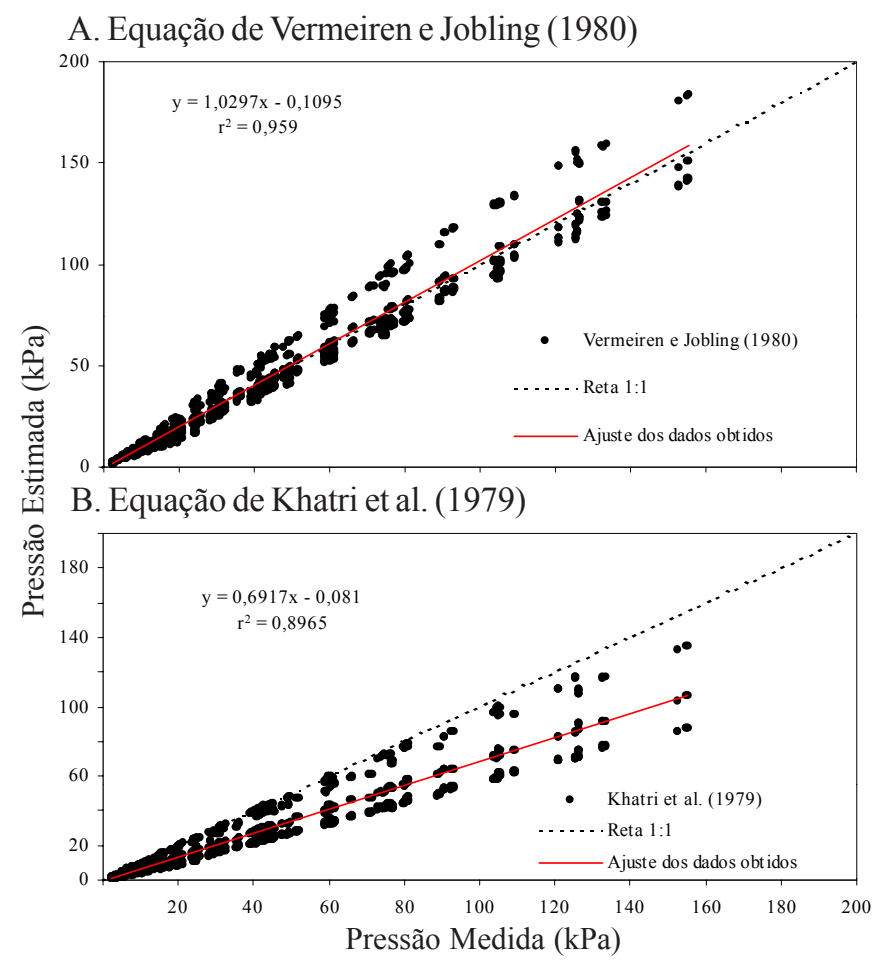

Figura 4. Relações entre pressões medidas e pressões estimadas, obtidas com base nas equações propostas por Khatri et al. (1979) e Vermeiren \& Jobling (1980)

uma função pressão medida versus pressão estimada próxima da reta $\mathrm{x}=\mathrm{y}$. Os valores de erro médio absoluto, obtidos na estimativa de pressão pelas equações de Vermeiren \& Jobling (1980) e Khatri et al. (1979) foram de 0,5 e 1,5 m respectivamente, valores elevados quando comparados com o valor do erro médio absoluto obtido pelo Modelo $3(0,16 \mathrm{~m})$, demonstrando novamente o desempenho destacado do Modelo 3.

\section{CONCLUSÕES}

1. Dentre os modelos propostos o que considerou a perda de carga localizada com o coeficiente $K$ da equação de perda carga localizada em função do Número de Reynolds (NR) foi o que melhor representou o fenômeno de perda de energia no microtubo.
2. As equações propostas por Vermeiren \& Jobling (1980) e Khatri et al. (1979) obtiveram um erro médio absoluto na estimativa de pressão de 0,5 e 1,5 m, respectivamente, valores elevados quando comparados com o erro médio obtido com o modelo que considera a perda de carga localizada com o coeficiente K em função do Número de Reynolds (0,16 m).

3. As estimativas dos diâmetros dos microtubos, com base nos dados experimentais, aproximaram-se dos valores medidos no Perfilômetro. Logo esta estimativa é uma alternativa viável, principalmente para quem não possui equipamentos especiais para medição do diâmetro do microtubo.

\section{LITERATURA CITADA}

Ishaq, A.M.; Ibrahim, M.A. The design of trickle irrigation systems with microtubes as emitters. In: International Congress of Microirrigation, 5, 1995, Orlando, Florida. Resumos... Orlando: ASAE, 1995, p.708-716.

Khatri, K.C.; Wu, I.; Giltlin, H.M.; Phillips, A. Hydraulics of microtube emitters. Journal of the Irrigation and Drainage Division of ASCE, New York, v.105, n. IR2, p.167-173, 1979.

Mackay, H. Low cost micro irrigation technologies for the poor. New Delhi: Department for Infrastructure and Urban Development, 2003. 37p. Affordable Micro Irrigation Technology Final Report.

Pizarro Cabello, F. Riegos localizados de alta frequencia. Madrid: Mundi-Prensa, 1987. 461p.

Porto, R.M. Hidráulica básica. 2. ed. São Carlos: EESC, 1999. $519 p$.

Silva, P.J.C.; Brito, R.A.L.; Azevedo, H.M. Caracterização hidráulica de linhas laterais em sistemas localizados utilizando microtubos de tamanhos variados como emissores. In: Congresso Nacional de Irrigação de Drenagem, 8, 1988, Florianópolis - SC. Resumos... Florianópolis: ABID, 1988, v.1, p.473-506.

Soares, A.A. Características hidráulicas de microtubos Cipla e linhas laterais para irrigação por gotejamento. Viçosa: UFV, 1981. 68p. Dissertação Mestrado.

Vermeiren, L.; Jobling, G.A. Localized irrigation. Design, installation, operation, evaluation. Rome: FAO, 1980. 203p. Irrigation and Drainage, Paper $n^{\circ} 36$. 